\title{
Editorial
}

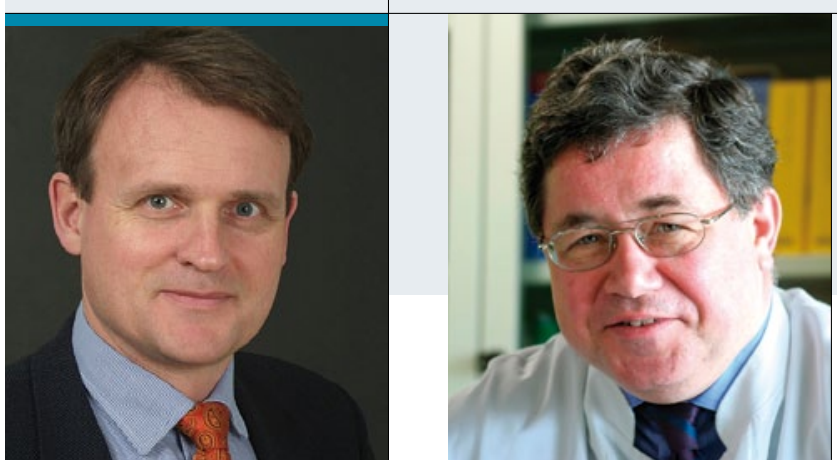

„Es wäre wünschenswert, wenn die im Rahmen des Präventionsgesetzes gesteckten Ziele auch im Bereich der Allergologie umgesetzt würden."

Prof. Dr. Thilo Jakob, Allergieabteilung \& Forschergruppe Allergologie, Universitäts-Hautklinik Freiburg Prof. Dr. Hans F. Merk, Hautklinik der Medizinischen Fakultät, Universitätsklinikum der RWTH Aachen

\section{Chance Präventionsgesetz nutzen!}

E nde März erreichte uns die erfreuliche Nachricht, dass das Bundeskabinett einen Gesetzesentwurf verabschiedet hat, der maßgeblich zur Verbesserung der Gesundheitsvorsorge führen soll. Ziel dieses Präventionsgesetzes soll u.a. sein, die Risiken durch Volkskrankheiten zu senken und die Eigenverantwortung der Patienten zu fördern, um chronische Erkrankungen zu verhindern. Hierfür sollen die Krankenkassen in die Pflicht genommen werden und für Präventionsleistungen insgesamt doppelt so viel ausgeben wie bisher. Die Kassen sollen besonders qualitätsgesicherte Präventionsprogramme finanzieren, d. h. Maßnahmen für die nachgewiesen wurde, dass sie nachhaltigen Erfolg bringen. Auch wenn die bisherigen Pressemitteilungen zu diesem Thema sich hauptsächlich auf die Volkskrankheiten Diabetes, Herz-Kreislauf-Erkrankungen und Depressionen beschränken, können und sollten wir diese Ziele auch für die Behandlung allergischer Patienten beanspruchen. Wir wissen um die präventive Wirksamkeit der spezifischen Immuntherapie in der Verhinderung des Etagenwechsels sowie in der Reduktion von Polysensibilisierungen. Diese Wirksamkeiten sind durch umfangreiche klinische Studien belegt. Trotz alledem zeigt der klinische Alltag, dass lediglich $40 \%$ der Patienten die einmal begonnene spezifische Immuntherapie auch über drei Jahre fortführen. Hier wäre es wünschenswert, wenn die im Rahmen des Präventionsgesetzes gesteckten Ziele auch im Bereich der Allergologie umgesetzt würden und die Leistungsträger dort Geld investieren, wo das volle Potenzial der spezifischen Immuntherapie als Präventionsmaßnahme tatsächlich noch nicht ausreichend ausgeschöpft ist. Ebenso sollte es Rückenwind geben für sekundäre Präventionsmaßnahmen die wir ja schon sehr erfolgreich im Bereich der Neurodermitis-, Asthma- und Anaphylaxieschulung durchführen. Wir hoffen sehr, dass das neue Präventionsgesetz auch zur Verbesserung der Situation unserer Allergiepatienten beiträgt.

Der eine oder andere von Ihnen wird sich über eine Beilage der Deutschen Akademie für Akupunktur, die den letzten beiden Ausgaben des Allergo
Journal beigefügt war, gewundert haben. Hier wurde zu einer Akupunktur-Fortbildungsveranstaltung eingeladen. Der Text beginnt mit der Aussage: „Das Leistungsvermögen der konventionellen Medizin in der Behandlung von Allergien ist unübersehbar eingeschränkt. Die Akupunktur hat sich dagegen immer mehr als eine gute effiziente und wirklich unverzichtbare Therapieoption herausgestellt." Unabhängig von der Frage der Wirksamkeit der Akupunktur in der Behandlung bestimmter allergischer Symptome möchten wir uns als Herausgeber des Allergo Journal von den Aussagen dieser Beilage distanzieren. Auch wir sind davon überrascht worden, denn es erfolgt a priori keine inhaltliche Prüfung von Anzeigen oder Beilagen durch die Herausgeber oder die Verlagsredaktion. Diese Abgrenzung in der Verantwortlichkeit spiegelt die in allen deutschen Pressegesetzen geforderte strikte Trennung von redaktionellen und werblichen Inhalten wider. Nichtsdestotrotz gibt es auf Seiten des Verlages Kontrollinstrumente, die jetzt wieder neu geeicht sind. Zum aktuellen Evidenzgrad für die Wirksamkeit der Akupunktur in der Behandlung der allergischen Rhinitis werden Sie in einer der nächsten Ausgaben eine Übersichtsarbeit vorfinden, die sich eingehend mit der Thematik befasst.

In der aktuellen Ausgabe haben wir einen CMEFortbildungsbeitrag zur nasalen Provokationstestung für Sie vorbereitet (S. 189). Zusätzlich finden Sie einen Übersichtsbeitrag zu dem häufig spät diagnostizierten Krankheitsbild der exogen-allergischen Alveolitis (S. 177). Abgerundet wird das Ganze durch eine Originalarbeit des Deutschen Polleninformationsdienstes zum Pollenflug in Deutschland in den Jahren 2001 bis 2011 (S. 168). Am Heftende erwartet sie schließlich ein Rückblick auf den Mainer Jubiläums-Allergie-Workshop im März (S. 205).

Wir wünschen Ihnen viel Freude bei der Lektüre der aktuellen Ausgabe und verbleiben mit herzlichen Grüßen.

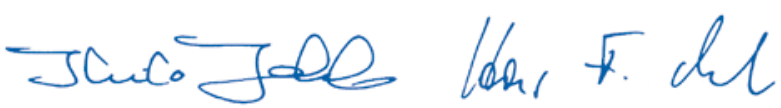

